\title{
IMPACTO DO PROGRAMA DE APOIO AO TABAGISTA DE UM MUNICÍPIO DO SUL DE MINAS GERAIS, BRASIL
}

\section{THE IMPACT OF THE SUPPORTING SMOKING CESSATION PROGRAM IN A COUNTY FROM THE SOUTH MINAS GERAIS, BRAZIL}

\section{IMPACTO DEL PROGRAMA DE APOYO AL FUMADOR EN UN MUNICIPIO DEL SUR DE MINAS GERAIS, BRASIL}

\author{
Sueli Leiko Takamatsu Goyatá* \\ Marcelo José Dias Silva \\ Walnéia Aparecida de SOUZA ${ }^{* * *}$ \\ Márcia Helena Miranda Cardoso Podestá ${ }^{* * *}$ \\ Luiz Alberto Beijo ${ }^{* * * *}$
}

\begin{abstract}
RESUMO
O objetivo deste estudo foi avaliar o impacto do Programa de Tratamento do Tabagismo de um ambulatório municipal, no sul de Minas Gerais, Brasil, na cessação de fumar. Foi realizada uma pesquisa qualitativa e quantitativa, descritiva e exploratória. Foram identificadas as características sociodemográficas, a história do tabagismo e a presença de comorbidades de 35 tabagistas, entrevistados nos domicílios. As entrevistas foram submetidas à análise de conteúdo. Em relação aos resultados quantitativos, 62,9\% dos usuários começaram a fumar antes dos 15 anos e 60\% apresentavam alta dependência. Em relação à cessação do tabagismo, 82,9\% pararam de fumar durante o ano de tratamento; após 18 meses, 58,6\% não apresentaram recaídas. $\mathrm{Na}$ análise qualitativa dos dados, foram identificadas oito categorias de análise: história tabágica; tabaco como forma de inclusão e de vínculo social; tabaco como fator de liberação das tensões da vida diária e de mecanismo compensatório; processo de mudança do hábito de fumar; conscientização do tabagismo como problema de saúde; fatores de sucesso para a cessação de fumar; recaída como fator de insucesso e cessação de fumar como resgate da autoestima. Este estudo demonstra a importância da implantação de programas anti-tabagismo pelo governo municipal, resultando em grande impacto na cessação do hábito de fumar dos usuários dos serviços públicos de saúde.
\end{abstract}

Palavras chave: Hábito de fumar, tratamento, abandono do uso de tabaco, enfermagem psiquiátrica.

\begin{abstract}
The aim of this study was to evaluate the impact of the Tobacco Treatment Program at a community clinic in the south of Minas Gerais, Brazil, for smoking cessation. A qualitative and quantitative, descriptive and exploratory research was conducted. Were identified sociodemographic characteristics, history of smoking and comorbidities

\footnotetext{
* Enfermeira. Professora da Escola de Enfermagem da Universidade Federal de Alfenas, Minas Gerais, Brasil. Email: sueligoyata@yahoo.com.br

${ }^{* *}$ Farmacêutico. Profissional residente em Saúde da Família da Universidade Federal de Alfenas, Minas Gerais, Brasil. Email: marcelo_farma05@yahoo.com.br

${ }^{* * *}$ Farmacêutica. Professora da Faculdade de Ciências Farmacêuticas da Universidade Federal de Alfenas, Minas Gerais, Brasil. Email: walne23@yahoo.com.br

${ }_{* * * \star}$ Farmacêutica. Professora da Faculdade de Ciências Farmacêuticas da Universidade Federal de Alfenas, Minas Gerais, Brasil. Email: mhmcardoso@hotmail.com

${ }^{* * * * *}$ Matemático. Professor do Instituto de Ciências Exatas da Universidade Federal de Alfenas, Minas Gerais, Brasil. Email: luizbeijo@yahoo.com.br
} 
of 35 smokers, interviewed in households. The interviews were subjected to content analysis. In relation to the quantitative results, $62.9 \%$ of users started smoking before 15 years and $60 \%$ had high dependency. Regarding smoking cessation, $82.9 \%$ had quit smoking during treatment and after 18 months, 58,6\% had no relapse. In qualitative data analysis eight categories were identified: smoking history; tobacco as a means of inclusion and social bonding; tobacco as the release factor from the stresses of daily living and compensatory mechanism, changing process of the smoking habit; awareness of smoking as a health problem; relapse as a failure factor and smoking cessation as a self-esteem release; successful factors for smoking cessation. This study demonstrates the importance of anti-smoking programs implementation by the municipal government, resulting in a significant impact on the cessation of smoking users of public health services.

Key words: Smoking, treatment, tobacco use cessation, psychiatric nursing.

\section{RESUMEN}

El objetivo de este estudio fue evaluar el impacto del Programa de Tratamiento del Tabaquismo de una clínica municipal, en el sur de Minas Gerais, Brasil, para dejar de fumar. Se realizó una investigación cualitativa y cuantitativa, descriptiva y exploratoria. Se identificaron las características sociodemográficas, antecedentes de tabaquismo y las comorbilidades de 35 fumadores, entrevistados en su casa. Las encuestas fueron sometidas al análisis del contenido. En relación a los resultados cuantitativos obtenidos, el 62,9\% de los usuarios comenzaron a fumar antes de los 15 años y el 60\% presentaba alta dependencia. En cuanto a dejar de fumar, el 82,9\% había dejado de fumar durante el tratamiento; después de 18 meses, el 58,6\% no presentaron recaídas. En el análisis cualitativo de los datos fueron identificados ocho categorías de análisis: historia de fumadores; tabaco como forma de inclusión y de vínculo social; tabaco como factor de liberación de las tensiones de la vida diaria y del mecanismo compensatorio; proceso de cambio em el hábito de fumar; concientización del tabaco como problema en la salud; fatores de éxito para dejar de fumar; recaída como factor de fracaso y cesación de fumar como rescate de la autoestima. Este estudio demuestra la importancia de la aplicación de los programas de lucha contra el tabaquismo implementado por el gobierno municipal, resultando un gran impacto sobre el abandono del consumo de tabaco de los usuarios de los servicios de salud pública.

Palabras clave: Hábito de fumar, tratamiento, cese del uso de tabaco, enfermería psiquiátrica.

Fecha recepción: 19/12/12 Fecha aceptación: 31/01/14

\section{INTRODUÇÃO}

O tabagismo é a principal causa global prevenível de morbidade e de mortalidade. Estimativas apontam que, em 2015, o tabaco será responsável por $10 \%$ dos óbitos globais e se projetam mais de oito milhões de óbitos para 2030, dos quais $83 \%$ ocorrerão nos países emergentes (1). Dados recentes indicam que os custos atribuíveis ao tabagismo são responsáveis por perdas de US\$ 500 bilhões ao ano devido à redução da produtividade, ao adoecimento e a mortes prematuras (2).

Atualmente, o tabagismo é amplamente reconhecido como uma doença epidêmica resultante da dependência de nicotina e classificado pela Organização Mundial de Saúde (OMS) no grupo dos transtornos mentais e de comportamento decorrentes do uso de substâncias psicoativas (3). Essa dependência faz com que os fumantes se exponham continuamente a cerca de 4.720 substâncias tóxicas, fazendo com que o tabagismo seja fator causal de aproximadamente 50 doenças diferentes, destacando-se as doenças cardiovasculares, o câncer e as doenças respiratórias obstrutivas crônicas $(4,5)$.

Segundo o Ministério da Saúde, dos cerca de $80 \%$ dos fumantes que desejam parar de fumar, apenas 3\% a cada ano conseguem sucesso (6). A baixa porcentagem de fumantes 
que conseguem parar de fumar indica que é necessária a atuação de profissionais especializados e de técnicas eficazes para o tratamento do tabagismo.

Nessa tarefa de ajudar os usuários a vencer a dependência do cigarro, podem ser priorizados alguns métodos de atuação. Há métodos que destacam o papel dos fármacos no tratamento, principalmente os considerados de primeira linha (que incluem a terapia de reposição de nicotina e a utilização de bupropiona) conjuntamente com a terapia comportamental breve em grupo ou individual (7).

Para enfrentar essa situação, o Ministério da Saúde assumiu, por meio do Instituto Nacional do Câncer, em 1989, o papel de organizar o Programa Nacional de Controle do Tabagismo. Esse Programa tem como objetivo reduzir a prevalência de fumantes no país e a consequente morbimortalidade por doenças tabaco-relacionadas. Para isso, utiliza as seguintes estratégias: prevenção da iniciação ao tabagismo; proteção da população contra a exposição ambiental à fumaça de tabaco; promoção e apoio à cessação de fumar e regulação dos produtos de tabaco, que visam ações educativas e de mobilização de políticas e de iniciativas legislativas e econômicas (6).

Dado o impacto mundial da dependência ao tabagismo sobre a saúde da população, diversos centros de cessação do tabagismo têm sido criados no Brasil. A Secretaria Municipal de Saúde de Alfenas-MG, por meio da equipe de saúde de um ambulatório municipal, implantado desde agosto de 2009, segue o modelo do Instituto Nacional do Câncer - INCA (8), o qual busca desenvolver várias atividades para o controle do tabagismo.

Conhecer o perfil dos tabagistas que manifestaram desejo próprio de cessar de fumar pode indicar os fatores associados ao sucesso terapêutico, especialmente de programas de apoio, tais como tempo de tabagismo, tempo de tratamento, idade de início e pontuação na Escala de Fagerström (9). Além disso, o levantamento das dificuldades pessoais e sociais associadas ao parar de fumar tem levado pesquisadores a conhecer os motivos que levaram à procura de apoio terapêtico, como conseguiram cessar de fumar, quais os problemas que enfrentaram ao parar, como os profissionais e as relações familiares ajudaram nesse período crítico e como perceberam os benefícios da cessação.

O objetivo deste estudo foi avaliar o impacto do Programa "Alfenas Livre do Tabaco" de um ambulatório municipal, no sul de Minas Gerais, Brasil, na cessação de fumar.

\section{Marco referencial}

Durante séculos, o uso do tabaco foi difundido mundialmente como erva dotada de propriedades medicinais capazes de curar diversas doenças. O comportamento de fumar foi tornando-se familiar e o cigarro, objeto de desejo de milhares de pessoas, especialmente a partir da década 1950, quando as técnicas de publicidade se desenvolveram com maior abrangência (6). Após 1960, surgiram os primeiros relatórios científicos que relacionaram o cigarro ao adoecimento do fumante. Apesar dos malefícios que o hábito de fumar provoca, a nicotina é uma das drogas mais consumidas do mundo. No Brasil, as manifestações pelo controle do tabagismo tornaram-se mais evidentes a partir da década de 1970 por iniciativa, principalmente, de organizações não governamentais. Somente no final da década de 1980, o Ministério da Saúde passou a formular políticas de controle ao tabagismo, com a criação do Programa Nacional de Controle do Tabagismo (10). De acordo com estudos do Ministério da Saúde, em 1994 havia 44,3 milhões de fumantes, cerca de $28 \%$ da população brasileira. Em 2010, esse número foi reduzido para 27,8 milhões, alcançando 14,5\% dos brasileiros. Essa redução deve-se tanto às campanhas públicas antitabagistas como à inflação do preço do cigarro $(11,12)$.

O tabagismo, antes visto como estilo de 
vida é atualmente reconhecido como um importante fator de adoecimento. A nicotina, principal droga lícita presente no cigarro, causa dependência física, química e psicológica, interferindo no organismo e no comportamento dos usuários. As consequências incluem efeitos destrutivos em vários tecidos, como doenças pulmonares, alterações celulares que predispõem às neoplasias malignas, assim como alterações cardíacas e vasculares (13). Por isso, a necessidade de investir em ações para apoiar as pessoas a deixaram de fumar é cada vez mais evidente (14).

A literatura mostra diferenças no padrão de consumo do tabaco em relação ao gênero e à classe social. Há um predomínio de fumantes do gênero masculino e se observa um crescimento de tabagistas em países em desenvolvimento, principalmente na população de baixa renda e de baixa escolaridade $(11,15)$. Estudo descrito por Cavalcante e Pinto (16) mostrou que muitos chefes de famílias de baixa renda utilizam parte de seus recursos financeiros para a compra de cigarros. De acordo com esses autores, o desgaste econômico é muito grande, envolvendo indivíduos, serviços e a sociedade.

Apesar da visível redução de fumantes a partir da década de 1990, no país, ainda se estima que aproximadamente 200 mil mortes por ano ainda ocorram em consequência do tabagismo.

No Brasil, o tratamento do tabagismo encontra-se disponível pelo Sistema Único de Saúde (SUS), por meio do Programa de Controle de Tabagismo, que oferece aconselhamento médico, tratamento farmacológico, terapia cognitivo-comportamental e terapia de grupo (17).

\section{MATERIAL E MÉTODO}

Foi realizado um estudo quali-quantitativo, descritivo e exploratório, com o objetivo de avaliar o impacto do Programa "Alfenas Li- vre do Tabaco", na cessação do hábito de fumar, durante o período de fevereiro de 2010 a agosto de 2011, em um ambulatório municipal de saúde. A pesquisa que integra tanto a abordagem qualitativa como a quantitativa é uma estratégia de investigação que utiliza a combinação de métodos e técnicas, a fim de se combinarem para alcançar os objetivos desejados. Cada etapa deve ser valorizada em sua singularidade, pois dessa valorização depende o sucesso da avaliação (18).

A população era constituída por 45 indivíduos cadastrados no Programa, residentes em quatro diferentes regiões de saúde do município de Alfenas. Considerando-se 2\% de erro amostral e respeitando-se a questão de gênero foram selecionados 35 indivíduos, adotando-se a amostragem por conglomerados.

Os tabagistas cadastrados no Programa passam a participar semanalmente das reuniões em grupo, conduzidas pela equipe de saúde multiprofissional (pneumologista, cirurgião dentista, enfermeiro, nutricionista e psicólogo). As reuniões no ambulatório municipal têm duração de uma hora e meia e média de seis sessões semanais, divididas ao longo de um ano. Nessas sessões, são discutidas as dificuldades e as barreiras de cessação de fumar, os fatores relacionados à dependência da nicotina e as alternativas de tratamento, que consistem em tratamento farmacológico, cuja medicação é fornecida gratuitamente, e não farmacológico. Ao término das sessões, o usuário é convidado a comparecer mensalmente ao ambulatório, até completar um ano da interrupção do tabagismo, como manutenção de seu tratamento.

Para fazer parte da pesquisa, estabeleceram-se como critérios de inclusão ser tabagista, ter idade maior de 18 anos, de ambos os sexos, cadastrados no Programa "Alfenas Livre do Tabaco" e ter assinado o Termo de Consentimento Livre e Esclarecido.

A coleta de dados quantitativos foi realizada pelo profissional residente em saúde da família por meio dos registros de dados secundários das fichas de cadastro, dos quais 
foram coletadas informações sobre as características sociodemográficas, razão que motivou a decisão de cessar o uso, a história tabágica, o grau de dependência pela Escada de Fagerström e a história de tentativas anteriores de cessar o uso. Essa escala auxilia na avaliação do grau de dependência à nicotina, com a seguinte pontuação: leve (0 a 4); médio (5 a 7) e alto (8 a 10) (9).

Os dados qualitativos foram coletados nos domicílios dos 35 indivíduos participantes do estudo, por meio de entrevista, após um período de 18 meses de finalização do programa. As entrevistas visavam ao levantamento sobre o desfecho do tratamento (cessação; redução; manutenção ou aumento do número de cigarros); se haviam cessado de fumar durante ou após a realização do grupo; se aderiram à medicação proposta e os motivos de não adesão; além da análise dos relatos e dos depoimentos dos participantes do estudo sobre as dificuldades pessoais e sociais associadas ao ato de parar de fumar. Nessa etapa da pesquisa, foi adotada a Análise de Conteúdo (19).

A análise de conteúdo, segundo Minayo (19), é dividida em três fases: a pré-análise; a exploração do material; o tratamento dos resultados obtidos e a interpretação. Na pré-análise, os documentos são analisados, segundo os objetivos iniciais da pesquisa. Nessa etapa são retomados os conceitos de forma categorizada para que direcione a análise final. $\mathrm{Na}$ exploração do material busca-se alcançar a compreensão dos relatos por meio das unidades de registro estabelecidas na pré-análise, classificando-as e as agregando em seguida. Na última fase os resultados obtidos são submetidos a uma análise de conteúdo formal dos discursos, sendo preservada a regularidade. A partir da análise de conteúdo, obtêm-se a apreensão da significação e a compreensão do objeto estudado (19).

Os resultados dos dados quantitativos foram apresentados em tabelas e analisados por meio de análise percentual das respostas encontradas e pelo tratamento estatístico. Os dados foram armazenados em banco de dados criado por meio do software SPSS for Windows versão 17.0 em que foram determinados a média e o desvio-padrão e, posteriormente, analisados por meio de Teste exato de Fisher, adotando-se nível de 5\% de significância $(\mathrm{p}<0,05)$.

Para a realização da pesquisa foi solicitada a concordância do usuário, na participação do estudo, mediante assinatura do Termo de Consentimento Livre e Esclarecido, sendo garantido o anonimato, o sigilo das informações prestadas pelos respondentes e a desistência em qualquer momento da pesquisa. Este estudo foi aprovado pelo Comitê de Ética em Pesquisa da Universidade Federal de Alfenas, Minas Gerais, sob o protocolo $\mathrm{N}^{\circ}$ 143/2011, de acordo com as Diretrizes e Normas Regulamentadoras de Pesquisa em Seres Humanos, Resolução do Conselho Nacional de Saúde (CNS) 196/96 (20).

\section{RESULTADOS}

Foram avaliados 35 tabagistas, com idade média de 42,14, DP 13,3 anos, variando de 25 a 65 anos. As características sociodemográficas da população estudada estão apresentadas na Tabela 1.

Houve predominância do gênero feminino $(80 \%)$, de estado civil solteiro $(42,9 \%)$ e de ensino fundamental incompleto (42,8 \%). A maioria dos participantes da pesquisa recebe até dois salários mínimos (62,9\%).

A Tabela 2 apresenta as características gerais dos tabagistas em relação ao tempo de consumo, às tentativas de cessação de fumar, às comorbidades e às razões da procura por tratamento. Houve predominância de usuários que iniciaram o tabagismo na faixa etária de 11 a 15 anos (62,9\%).

O tempo médio de tabagismo foi de 32 anos, $\mathrm{DP} \pm 12$ anos, sendo que a maioria (77\%) referiu tragar sempre a fumaça do cigarro, $17 \%$ tragavam às vezes ou nunca e $6 \%$ não responderam. 
Tabela 1. Características sociodemográficas dos tabagistas, Alfenas, 2012.

\begin{tabular}{lrc}
\hline Variáveis & N & $\%$ \\
\hline Gênero & 28 & 80 \\
Feminino & 7 & 20 \\
Masculino & & \\
\hline Estado Civil & 14 & 40 \\
Casado & 15 & 42,9 \\
Solteiro & 6 & 17,1 \\
Outros $^{*}$ & & \\
Grau de Escolaridade & 2 & 5,8 \\
Analfabeto & 15 & 42,8 \\
Ensino Fundamental Incompleto & 5 & 14,3 \\
Ensino Fundamental Completo & 12 & 34,3 \\
Ensino Médio Completo & 1 & 2,8 \\
Ensino Superior Completo & & \\
\hline Renda Mensal & 5 & 14,3 \\
< 1 Salário Mínimo** & 17 & 48,6 \\
1-2 Salários Mínimos & 9 & 25,7 \\
2-3 Salários Mínimos & 4 & 11,4 \\
$>$ 3 Salários Mínimos & & \\
\hline
\end{tabular}

${ }^{*}$ Divorciado, viúvo e separado.

**Valor do Salário Mínimo vigente: R\$622,00.

Tabela 2. Características gerais dos tabagistas, Alfenas, 2012.

\begin{tabular}{lrc}
\hline Variáveis & N & $\%$ \\
\hline Tabagismo & & \\
Começou a fumar antes de 10 anos de idade & 4 & 11,4 \\
Começou a fumar entre 11 e 15 anos de idade & 22 & 62,9 \\
Começou a fumar após 16 anos de idade & 9 & 25,7 \\
\hline Tentativas de cessação de fumar & & \\
Ao menos uma tentativa de cessar de fumar & 19 & 54,3 \\
Duas tentativas de cessar de fumar & 12 & 34,3 \\
Mais de três tentativas de cessar de fumar & 4 & 11,4 \\
\hline Grau de dependência & & \\
Leve & 6 & 17,1 \\
Médio & 8 & 22,9 \\
Alto & 21 & 60 \\
\hline Morbidades & & \\
Hipertensão Arterial & 11 & 31,4 \\
Ansiedade & 7 & 20 \\
Doença Respiratória & 7 & 20 \\
Diabetes mellitus & 5 & 14,3 \\
Depressão & 5 & 14,3 \\
\hline Motivos de procura por tratamento & \\
Preocupaçães com a saúde & & \\
Orientação médica & 26 & 74,3 \\
Pressão familiar & 10 & 28,6 \\
Pressão social ou no trabalho & 9 & 25,7 \\
Custo do cigarro & 3 & 8,6 \\
\hline * usaro podra apresentar & 1 & 3 \\
\hline
\end{tabular}

${ }^{\star} \mathrm{O}$ usuário poderia apresentar mais de um motivo. 
Em relação às comorbidades, $31,4 \%$ dos usuários apresentavam hipertensão arterial, seguida da ansiedade (20\%) e da doença respiratória $(20 \%)$.

A maioria dos usuários procurou o serviço voluntariamente devido à preocupação com a saúde $(74,3 \%)$. Neste estudo, apenas $3 \%$ dos usuários apresentaram o custo do cigarro como motivo para buscar tratamento.

O grau de dependência encontrado foi muito elevado (Fagerström $>6$ ), na maioria dos tabagistas, sendo que 21 (60\%) apresentaram alto grau de dependência o que cons- titui um grave problema. Dos 21 tabagistas com alto grau de dependência, 17 (58,6\%) cessaram de fumar durante o primeiro ano. Ao comparar os tabagistas de alta dependência com os de média e baixa dependência que cessaram de fumar, não houve diferença significativa ( $\mathrm{p}=0,0697)$. Os resultados encontram-se nas Tabelas 3 e 4.

O número de usuários que tinham recebido tratamento farmacológico durante as tentativas anteriores foi de 94 e $6 \%$ dos pacientes não fizeram nenhum uso de medicamentos.

Tabela 3. Distribuição percentual dos participantes segundo o grau de dependência, cessação de fumar no primeiro ano e recaída após 18 meses do término do programa.

\begin{tabular}{lcccccc}
\hline Variáveis & \multicolumn{2}{c}{ Grau de dependência } & \multicolumn{2}{c}{ Cessação de fumar } & \multicolumn{2}{c}{ Recaída } \\
\cline { 2 - 7 } & $\mathbf{N}$ & $\%$ & $\mathbf{N}$ & $\%$ & $\mathbf{N}$ & $\%$ \\
\hline Alto grau de dependência & 21 & 60 & 17 & 58,6 & 10 & 83,4 \\
Média dependência & 8 & 22,9 & 7 & 24,2 & 1 & 8,3 \\
Baixa dependência & 6 & 17,1 & 5 & 17,2 & 1 & 8,3 \\
Total & 35 & 100 & 29 & 100 & 12 & 100 \\
\hline
\end{tabular}

Tabela 4. Desfecho do programa antitabagista, Alfenas, 2012.

\begin{tabular}{|c|c|c|c|c|c|}
\hline \multirow{3}{*}{ Variáveis } & \multicolumn{4}{|c|}{ Cessou de fumar } & \multirow{3}{*}{ Valor-p } \\
\hline & \multicolumn{2}{|c|}{ Sim } & \multicolumn{2}{|c|}{ Não } & \\
\hline & $\mathbf{N}$ & $\%$ & $\mathbf{N}$ & $\%$ & \\
\hline \multicolumn{6}{|c|}{ Participação em sessões (seis) } \\
\hline Quatro a seis & 28 & 80 & 0 & 0 & \\
\hline Menos de quatro & 1 & 2,9 & 6 & 17,1 & \\
\hline Total & 29 & 82,9 & 6 & 17,1 & $<0,0095$ \\
\hline \multicolumn{6}{|c|}{ Apresentou recaída após 18 meses } \\
\hline Sim & 0 & 0 & 12 & 41,4 & \\
\hline Não & 17 & 58,6 & 0 & 0 & $<0,0001$ \\
\hline \multicolumn{6}{|c|}{ Grau de dependência } \\
\hline Alto & 17 & 48,6 & 4 & 11,4 & \\
\hline Médio ou baixo & 12 & 34,3 & 2 & 5,7 & \\
\hline Total & 29 & 82,9 & 6 & 17,1 & $<0,0697$ \\
\hline
\end{tabular}


Quanto à avaliação sobre o apoio da equipe de saúde do Programa "Alfenas Livre do Tabaco" recebido pelos usuários, 57,1\% afirmaram que tiveram apoio total da equipe durante o tratamento e $40 \%$, frequentemente. Quanto à qualidade do Programa oferecido aos usuários, $40 \%$ avaliaram como ótimo, $57,1 \%$, como bom e 2,9\%, não responderam.

A análise estatística mostra uma associação significativa entre as variáveis número de participação dos usuários nas sessões e a cessação de fumar $(p=0,0095)$. O mesmo foi verificado em relação ao número de participação nas sessões e não apresentar recaída após 18 meses do término do programa $(\mathrm{p}<0,0001)$. Tais resultados mostram que a participação do tabagista em quatro a seis sessões do programa parece ser decisiva para cessar de fumar. Esses resultados encontramse na Tabela 4.

Portanto, verificou-se que o Programa "Alfenas Livre do Tabaco" mostrou-se efetivo, após o término das sessões no primeiro ano uma vez que 29 (82,9\%) dos usuários tiveram cessação.

A análise de conteúdo teve início com a transcrição literal das entrevistas, focalizando a experiência vivida pelos entrevistados em sua passagem da condição de dependentes para abstinentes do tabaco e as diferentes etapas dessa trajetória. Após a transcrição, foi realizada a pré-análise; em seguida, a exploração do material e, finalmente, a identificação das categorias analíticas.

Foram identificadas oito categorias de análise: a) história tabágica; b) o tabaco como forma de inclusão e de vínculo social; c) o tabaco como fator de liberação das tensões da vida diária e de mecanismo compensatório; d) processo de mudança do hábito de fumar; e) conscientização do tabagismo como problema de saúde; f) fatores de sucesso para a cessação de fumar; g) recaída como fator de insucesso; h) a cessação de fumar como resgate da autoestima.

\section{a) História tabágica}

A iniciação no fumar foi narrada pela maioria dos usuários como tendo surgido na passagem da infância para a adolescência, favorecida pela convivência intensa com familiares fumantes na própria residência, havendo destaque para a figura do pai. Também na relação com amigos próximos, o fumar era compartilhado. O convívio com fumantes representava uma situação muito atrativa e inevitável para incentivar o hábito de fumar, conforme afirmaram alguns entrevistados:

Eu convivi a minha adolescência, com os meus amigos e familiares, todos fumando. (Entrevistado 1)

Meu pai fumava [...] até quando estava doente. Foram anos fumando cigarro de palha. (Entrevistado 8)

Convivi com amigos, no emprego. Sempre você convive com fumantes e é difícil separar. (Entrevistado 12)

\section{b) O tabaco como forma de inclusão e de vínculo social}

O tabagismo como um fator de inclusão social e o compartilhar o tabaco contribuía para o estabelecimento do vínculo social:

No meu tempo, que eu comecei a fumar, eu comecei a fumar eu tinha dezessete anos, era chique. Se não fumasse, eu estava excluída da roda dos meus amigos. (Entrevistado 12)

Eu fumava e achava importante perante meus colegas e a sociedade [...] bonito o cigarro entre os dedos, pessoa importante [...] muitos me viam como autoridade, me respeitavam. (Entrevistado 6)

\section{c) O tabaco como fator de liberação das ten- sões da vida diária e como mecanismo compensatório}

Alguns usuários narraram que o fumar estava intimamente relacionado ao estar só e aos 
estados de ansiedade:

Tinha vontade de fumar quando eu estava sozinha, depressiva, nervosa, preocupada com alguma situação, neste momento fumava vários cigarros. (Entrevistado 22)

Quando estava irritado, nervoso, fumava bastante [...] o cigarro me acalmava. Quando eu estava trabalhando, passava até horas sem fumar. (Entrevistado 33)

\section{d)Processo de mudança do hábito de fumar}

O sabor deixado pelo cigarro na boca foi relatado como percebido a partir de um determinado momento, de modo "diferente" e, assim, o fumar deixava de ser prazeroso.

Fumava porque minha colega fumava. $O$ cheiro me atraía. E depois foi o cheiro do cigarro que me fez parar de fumar, ficava enjoada. (Entrevistado 5)

No inicio [...] o cigarro tinha diferente sabor, hoje em dia o sabor tá diferente, mais desagradável, foi aí que percebi [...] às vezes, eu fumava, não tava agradando mais. (Entrevistado 9)

Meu marido me disse que, quando me beijava, parecia que estava beijando um cinzeiro. (Entrevistado 24)

\section{e) Conscientização do tabagismo como problema de saúde}

A participação dos usuários nas sessões possibilitou a reelaboração cognitiva de seus conceitos sobre os problemas relacionados à saúde devido ao tabagismo. Houve uma mobilização para os relatos sobre suas doenças e dificuldades para a cessação de fumar, o que os levou a identificarem o tabagismo como um problema a ser enfrentado individual e coletivamente. A cessação do tabagismo decorreu de um aprendizado que lhes proporcionou a compreensão do problema em suas diferentes, porém interrelacionadas dimensões, ou seja: na dimensão do sujeito, na de sua relação com as outras pessoas e em sua inserção social:

Eu acho que foi a autoanálise sobre o que é realmente o cigarro, tudo que era explicado nas reuniões [...] graças o apoio deste programa, eu não voltei a fumar. (Entrevistado 5)

...o que me ajudou parar de fumar mesmo, foi quando eu vi no grupo de tabagismo, relatos de pessoas que há anos estão tentando largar de fumar e não conseguiu. [...] eu achei que chegou o momento certo e que o cigarro não ia me dominar [...]. (Entrevistado 19)

Na quarta sessão em diante, eu já sentia, que tinha condições de parar de fumar. (Entrevistado 28)

\section{f) Fatores de sucesso para a cessação de fumar}

O tratamento realizado pelo Programa foi percebido pelos usuários como o preparo para a cessação de fumar e para a manutenção dessa abstenção. Nos encontros em grupo, os participantes compartilharam experiências de sucesso. Os entrevistados, em geral, relatam um melhor relacionamento com os familiares devido ao fato de cessarem de fumar. A diminuição do paladar, o mau cheiro no corpo, o constrangimento de fumar em público, por não ocorrerem mais, são vistos pelos usuários como sendo os ganhos e os fatores para seguirem adiante na abstenção:

E hoje, quando vejo um fumante, o que vejo é aquela pele seca, cabelo com mau cheiro, pigarro [...] eu era assim [...] eu ficava com vergonha. (Entrevistado 5)

Nos primeiros seis meses, aconteceu isso três vezes [...] eu cheguei a pegar o cigarro [...] quando eu vi ele, eu falei comigo: não posso e não posso. (Entrevistado 2)

Esse programa que é ótimo veio na hora certa na minha vida. No grupo, eu relatada os problemas, meus colegas e o pessoal da equipe me entendiam. Hoje, sei o mal que passei, agora sinto até o meu perfume. (Entrevistado 24) 


\section{g) Recaída como fator de insucesso}

Como experiência de insucesso, evidencia-se a recaída. Os participantes que voltaram a fumar, após as sessões de tratamento, se sentem fracassados e lutam constantemente para parar de fumar:

Voltei a fumar por falta de alguma coisa na minha vida, me sinto um vazio [...] o programa foi ótimo, mas, com o passar do tempo, senti a necessidade de voltar, acho que foi com motivos econômicos e familiares. (Entrevistado 14)

O vício domina a pessoa, mesmo, tenho certeza, domina. Depende muita da pessoa que fuma, se não tiver força de vontade, ele nunca vai largar a parar de fumar [...] e ele me dominou não tive a força de vontade que muitos dos meus colegas tiveram. (Entrevista 33)

\section{h)A cessação de fumar como resgate da au- toestima}

O parar de fumar significou para alguns participantes, além do resgate da autoestima, a possibilidade de recuperar o vínculo nas relações com familiares e com amigos. Esses participantes relataram que não eram condizentes com os princípios de respeito ao próximo:

Hoje, percebo o quanto eu prejudiquei um tanto de gente, fumando, por exemplo, no ambiente do meu trabalho. Chegava a qualquer lugar e logo já ia fumando. (Entrevistado 10)

...meus filhos, meu marido ajudaram-me muito, porque eles reclamavam do cheiro, fumaça e dos cigarros pela casa. Hoje sou outra pessoa, mais disposta. (Entrevistado 7)

Hoje, vejo que não é preciso fumar para ter amigo. Vivo intensamente sem esse mal. Hoje o meu irmão, minhas filhas me admiram e me sinto valorizado. (Entrevistado 35)

\section{DISCUSSÃO E CONCLUSÃO}

A maioria dos tabagistas que procurou atendimento para o tratamento da dependência à nicotina era do gênero feminino. A predominância de fumantes de mulheres entre os ingressantes em programas de cessação do tabagismo foi também verificada em outros estudos nacionais. Em pesquisa realizada em Fortaleza (CE), observou-se predominância de $65,6 \%$ (21) e de 62\%, em estudo realizado na cidade de Porto Alegre (RS) (22). Essa predominância pode ser justificada pelo fato de as mulheres exibirem maiores cuidados com a saúde do que os homens. Além disso, muitas delas não tinham compromissos profissionais, o que facilitava o comparecimento às sessões do grupo de apoio (23).

Em Portugal e nos países ocidentais, em geral, o início ao tabagismo também ocorre na faixa etária entre 11 a 15 anos (24). Diversos estudos têm demonstrado que o tabagismo tem início entre o final da infância e da jovem adolescência, quando o mesmo não é percebido como um risco para a saúde (25).

Quanto mais cedo ocorrer a iniciação, mais grave será a dependência, maior será a dificuldade para deixar de fumar, mais longo será o percurso de fumante e mais graves serão os danos à saúde. $\mathrm{O}$ uso de tabaco na adolescência associa-se também ao consumo de outras drogas, a outros problemas de comportamento, a perturbações emocionais e a dificuldades relacionais (26).

No Brasil, estudos mostraram que o alto grau de dependência à nicotina está associado ao maior risco de fracasso no tratamento (27).

Segundo evidência epidemiológica disponível, existe uma relação de causalidade entre o tabagismo e cerca de 50 doenças, entre as quais se destacam a cardiovascular, o câncer e as doenças respiratórias (1). O fumo também é responsável por $18 \%$ das mortes por câncer em países em desenvolvimento. Entre os tipos de câncer cuja associação é bem es- 
tabelecida com o tabagismo, destacam-se os cânceres de pulmão, de laringe e de esôfago (2).

O percentual de indivíduos que deixaram de fumar ao término de participação no programa e após 18 meses é relevante, não apenas para os tabagistas, como também para os familiares e profissionais de saúde, especialmente para aqueles que apresentavam alto grau de dependência à nicotina. Tais resultados impactaram na rede de serviços de saúde do município, com a expansão e com a implantação do Programa "Alfenas Livre do Tabaco" em novas unidades. No Brasil, esse programa revela a importância de uma política de governo federal descentralizado e da corresponsabilidade do poder público municipal no controle do tabagismo. Pesquisas mostram que cerca de $80 \%$ dos fumantes desejam parar de fumar. No entanto, apenas 3\% conseguem a cada ano, e a maioria desse grupo cessa sem ajuda. Esse cenário se modifica quando programas de governo são implantados nas redes de atenção primária à saúde, elevando as taxas de cessação de fumar de $3 \%$ para 20 a 30\% em um ano (28).

Estudo prospectivo realizado no Reino Unido, com duração de 12 anos, com cerca de 1,2 milhões de mulheres, apresentando idade média de 55 anos, mostra que fumantes no início da pesquisa apresentaram uma relação de mortalidade quase três vezes maior em relação às não fumantes. Parar de fumar antes do 40 anos de idade evita mais de $90 \%$ do excesso de mortes provocado pelo tabagismo. Os resultados comprovam os benefícios da cessação do hábito de fumar (29).

Em geral, os dependentes de drogas, para suportar as tensões geradas das resoluções insatisfatórias dos problemas cotidianos, lançam mão dessas substâncias, como uma compensação acessória, como um substituto para seus recursos internos para enfrentar, de maneira proveitosa, os acontecimentos que marcam suas vidas. Com a droga, acreditam que poderão atingir um estado de equilíbrio e uma resolução satisfatória da tensão (30).
Entre as razões que os usuários relatam para continuar fumando, está o alívio dos sintomas de ansiedade e de depressão. Além disso, sabe-se que a ansiedade está associada ao risco aumentado de lapso durante a cessação do tabagismo e os sintomas depressivos são preditores significativos de lapsos e de recaídas precoces (31).

Neste estudo, houve predominância de participantes com ensino fundamental incompleto, baixa escolaridade e baixa renda, que são variáveis associadas ao tabagismo. Em um estudo realizado na China, verificouse que indivíduos com baixa escolaridade têm uma probabilidade cerca de sete vezes maior de serem fumantes do que indivíduos com nível superior e, no Brasil, essa probabilidade é cinco vezes maior (22). As estratégias para a expansão do mercado da indústria do tabaco que estimulam o consumo e facilitam o acesso ao cigarro contribuem para o seu maior consumo pelas populações de baixa renda e escolaridade (32).

Aspectos econômicos relacionados ao tabagismo são descritos no estudo realizado por Caram et al. (33), no qual se avaliou a importância de políticas econômicas na redução do consumo do tabaco, mostrou-se que metade dos fumantes relatou mudanças no comportamento em relação ao tabagismo em consequência do aumento do preço do cigarro.

O perfil da clientela atendida pelo Programa, em sua maioria de baixa renda, mostra a importância do acesso dos usuários aos serviços públicos de saúde e da disponibilidade de recursos farmacológicos gratuitos. As abordagens farmacológicas e não farmacológicas são efetivas para aumentar a taxa de cessação do tabagismo e a associação das duas formas de tratamento fornece os melhores resultados (34). A disponibilidade de medicação pelos serviços públicos deve ser, portanto, ampliada para aumentar a taxa de abstinência e quebrar esse círculo vicioso.

Os resultados apontam como fator de sucesso na cessação de fumar a participação dos 
tabagistas em quatro a seis sessões do Programa "Alfenas Livre do Tabaco". Para tanto, parece ser fundamental o desenvolvimento de ações sistematizadas desse Programa para se manter um fluxo contínuo de informações e de sensibilização dos usuários.

A proposta de auxiliar os usuários na cessação de fumar constitui-se um grande desafio, exigindo acompanhamento sistematizado pelo grupo terapêutico, capacitação e criatividade por parte dos profissionais de saúde. Considera-se que culturas e hábitos são passíveis de mudança somente em longo prazo. A inclusão e a ampliação de estratégias motivacionais precisam ser consideradas no processo que antecede a tentativa de cessação de fumar, principalmente em serviços públi$\cos (32)$.

Esse Programa, portanto, tem utilizado canais de comunicação para alcançar a comunidade de forma contínua e dentro da realidade de sua rotina. Isso reforça a importância de se conhecer o perfil da clientela atendida pelo Programa para a avaliação das estratégias empregadas e para a adequação das propostas de tratamento para tabagistas, visando ao aumento das taxas de cessação de fumar e à redução dos índices de recaída. Com isso, diminuem-se principalmente as taxas de internações e de mortes por doenças cardiovasculares, por neoplasias e por doenças respiratórias em adultos.

Agradecimentos: Os autores agradecem a Universidade Federal de Alfenas, pelo apoio institucional; a Secretaria Municipal de Saúde de Alfenas, pela disponibilidade de cenários de práticas e o Ministério da Saúde, pelo apoio financeiro para o bolsista do Programa de Residência.

\section{REFERÊNCIAS}

1. Pinto MM, Ugá AD. Custo do tratamento de pacientes com histórico de tabagismo em hospital especializado em câncer. Rev Saude Publica. 2011; 45(3): 575-82.

2. Shafey O, Eriksen M, Ross H, Mackay J. The Tobacco Atlas. 3a ed. Atlanta: American Cancer Society; 2009.

3. Organização Mundial de Saúde (OMS). The World Health Report: Reducing Risks and, Promoting Healthy Lifestyles. Geneva; 2002.

4. Rosemberg J. Pandemia do Tabagismo Enfoques Históricos e Atuais. São Paulo: Secretaria Estadual de Saúde; 2002.

5. World Health Organization. Building blocks for tobacco control: a handbook [Internet]. Geneva: WHO; 2004 [citado 01 maio 2011]. Disponível: <http://www. who.int/tobacco/resources/publications/ tobaccocontrol_handbook/en/

6. Ministério da Saúde Brasil. Instituto Nacional de Câncer (INCA). Abordagem e Tratamento do Fumante - Consenso. Rio de Janeiro: INCA; 2001.

7. Marques ACPR, Campana A, Gigliotti AP, Lourenço MTC, Ferreira MP, Laranjeira R. Consenso sobre o tratamento da dependência de nicotina. Rev Bras Psiquiatr. 2001; 23(4): 200-14.

8. Instituto Nacional do Câncer (INCA). Plano de implantação da abordagem e tratamento do tabagismo na Rede SUS. Fluxos de Informação e Instrumentos de Avaliação Manual de Operação. Rio de Janeiro: INCA; 2009.

9. Fagerström KO, Schneider NG. Measuring nicotine dependence: a review of the Fagerström Tolerance Questionnaire. J Behav Med. 1989; 12(2): 159-82.

10. Danel I, Kurowski C, Saxenian H. 2005. Brazil - Addressing the Challenge of Non Communicable Diseases. Washington DC: The World Bank; 2005.

11. Ministério da Saúde Brasil. Secretaria de Vigilância em Saúde. Instituto Nacional do Câncer. Coordenação de Prevenção e Vigilância. Inquérito Domiciliar sobre Comportamentos de Risco e Morbidade Referida de Doenças e Agravos não 
Transmissíveis. Brasil, 15 capitais e Distrito Federal 2002-2003. Rio de Janeiro: INCA; 2004.

12. Mirra AP, Meirelles RHS, Godoy I. et al. Diretrizes em foco. Tabagismo - Parte I. Rev Assoc Med Bras. 2010; 56(2): 127-43.

13. Chaves EC, Mendonça LGT. Uso e dependência do tabaco. En: Benseñor IFCT, Silveira MM, Silva FMS, Dorea EL, Lotufo PA, organizadores. Medicina em ambulatório: diagnóstico e tratamento. São Paulo: Sarvier; 2006.

14. Ministério da Saúde Brasil/Instituto Nacional de Câncer (INCA). 2003 - Legislação Federal sobre Tabaco no Brasil, atualizado em agosto de 2003. Brasília: MS; 2003.

15. Ministério da Saúde Brasil. VIGITEL Brasil 2006. Vigilância de Fatores de Risco e Proteção para Doenças Crônicas por Inquérito Telefônico. Brasília: MS; 2006.

16. Cavalcante TM, Pinto M. Considerações sobre tabaco e pobreza no Brasil: consumo e produção de tabaco. [Internet] INCA: 2005. [citado 16 março de 2013]. Disponível em: www.inca.gov.br/tabagismo/publicacoes/tabaco_pobreza.pdf.

17. Instituto Nacional do Câncer (INCA). Coordenação de Prevenção e Vigilância Comprev. Deixando de Fumar sem Mistério. Rio de Janeiro: MS/INCA, 2004.

18. Minayo, MCS. Avaliação por triangulação de métodos: abordagem de programas sociais. Rio de Janeiro: Fiocruz; 2005.

19. Ministério da Saúde Brasil. Minayo, MCS. O desafio do conhecimento: pesquisa qualitativa em saúde. $7^{a}$ ed. São Paulo: Hucitec-Abrasco; 2000.

20. Ministério da Saúde Brasil. Conselho Nacional de Saúde/Comitê Nacional de Ética em Pesquisa em Seres Humanos. Resolução na 196 , de 10 de outubro de 1996. Diretrizes e Normas regulamentadoras de pesquisa envolvendo seres humanos. Brasília: Ministério da Saúde; 1996.

21. Sales MP, Figueiredo MR, Oliveira MI, Castro HN. Outpatient smoking cessa- tion program in the state of Ceará, Brazil: patient profiles and factors associated with treatment success. J Bras Pneumol. 2006; 32(5): 410-7.

22. Balbani APS, Montovani JC. Métodos para abandono do tabagismo e tratamento da dependência de nicotina. Rev Bras Otorrinolaringol. 2005; 71(6): 82-7.

23. Karen SKK, Oliveira ML, Pádua AI, Vieira F, Martinez JAB. Características clínicas de fumantes atendidos em um centro de referência na cessação do tabagismo. Medicina (Ribeirão Preto) 2012; 45(3): 337-42.

24. Vitória PD, Silva AS, Vries HD. Avaliação longitudinal de programa de prevenção do tabagismo para adolescentes. Rev Saude Publica. 2011; 45(2): 343-54.

25. Moura MAS, Menezes MFB, Mariano RD, Silva VR, Sousa LP. Intervenções de Enfermagem no Controle do Tabagismo: uma Revisão Integrativa. Rev Brasil Câncer. 2011; 57(3): 411-419.

26. Iglesias V, Cavada G, Silva C, Cáceres D. Consumo precoz de tabaco y alcohol como factores modificadores del riesgo de uso de marihuana. Rev Saude Publica. 2007; 41(4): 517-22.

27. Chatkin JM, Mariante AC, Haggsträm FM, Wagner MB, Fritscher CC. Abstinence rates and predictors of outcome for smoking cessation: do Brazilian smokers need special strategies? Addiction. 2004; 99(6): 778-84.

28. Brasil. Ministério da Saúde. Instituto Nacional do Câncer. Abordagem e tratamento do fumante - Consenso 2011. Rio de Janeiro: INCA; 2001.

29. Pirie K, Peto R, Reeves GK, Green J, Beral V. The Lancet 2013; 381 (9861): 133-141.

30. Kalina E. Drogradição hoje: indivíduo, família e sociedade. Porto Alegre: Artmed; 2008.

31. Lima MS, Viegas CAA. Avaliação do Grau de Ansiedade, Depressão e Motivação dos Fumantes que Procuraram Tratamento para Deixar de Fumar no Distrito Fede- 
ral. Rev Brasil Câncer. 2011; 57(3): 345353.

32. Azevedo RCS, Horihiga CM, Assumpção ISMA, Frazatto CRG. Grupo terapêutico para tabagistas: resultados após seguimento de dois anos. Rev Assoc Med Bras. 2009; 55(5): 593-6.

33. Caram, LMO, Ferrari R, Tanni SE, Coelho
LS, Godoy I, Martin RSS. Characteristics of smokers enrolled in a public smoking cessation program. J Bras Pneumol. 2009; 35(10): 980-85.

34. Le Foll B, George TP. Treatment of tobacco dependence: integrating recent progress into practice. CMAJ. 2008; 178(6): 732. 